\title{
Texture Analysis for Stroke Classification in Infrared Reflectogramms
}

\author{
Martin Lettner and Robert Sablatnig \\ Vienna University of Technology, \\ Institute of Computer Aided Automation, \\ Pattern Recognition and Image Processing Group, \\ Favoritenstrasse 9/183/2, 1040 Vienna, Austria \\ \{lettner, sab\}@prip.tuwien.ac.at \\ http://www.prip.tuwien.ac.at
}

\begin{abstract}
The recognition of painted strokes is an important step in analyzing underdrawings in infrared reflectogramms. But even for art experts, it is difficult to recognize all drawing tools and materials used for the creation of the strokes. Thus the use of computer-aided imaging technologies brings a new and objective analysis and assists the art experts. This work proposes a method to recognize strokes drawn by different drawing tools and materials. The method uses texture analysis algorithms performing along the drawing trace to distinguish between different types of strokes. The benefit of this method is the increased content of textural information within the stroke and simultaneously in the border region. We tested our algorithms on a set of six different types of strokes: 3 classes of fluid and 3 classes of dry drawing materials.
\end{abstract}

\section{Introduction}

Infrared reflectogramms are a popular tool for the investigation of underdrawings from medieval painted works of art. Underdrawings constitute the basic concept of an artist when he starts his work of art. Normally they are hidden by paint layers of the finished work and thus unseen in the visible range of the electromagnetic spectrum. Infrared reflectography (IRR) allows a look through the paint layers and thus a visualization of the underdrawing. The wavelength of IRR lies in the range from approx. 1000 to $2500 \mathrm{~nm}$ where the longer wavelength facilitates the penetration of the paint layers. The generated image is called infrared (IR) reflectogramm [3]. Conservators and art historians are interested in the development of underdrawings, their relation to other drawings and differences between underdrawings and the covering painting. Further more painting instructions and the identification of the drawing tool and material used for the creation of the underdrawing are of particular interest. But the recognition of the drawing tool and material of painted strokes in IR images is not always clear and unambiguous. The limited resolution of the acquisition system, the use of different tools in a painting and disturbing paint layers make a recognition with the naked eye difficult. Thus the use of computer-aided systems can assist art 
experts in doing their work comparable to the usage of computers in medical applications which are nowadays inconceivable without computers.

Painted Strokes can be drawn either in dry or fluid drawing material. Chalk and graphite are examples for dry materials and paint or ink applied by pen or brush are examples for fluid painting materials. The appearance of the boundary characteristics, the texture, the stroke endings or the color variety can be used for the visual recognition.

In this work we are going to develop an algorithm which allows the identification of the drawing material used for the creation of painted strokes. Several work in this direction has been done before. A segmentation and classification of underdrawing strokes by the help of snakes is reported in [6]. The analysis of the texture of strokes is shown in [4] and [8]. Wirotius et al. [14] showed a differentiation in gray level distributions for writer identification.

Our approach differs with respect to [4] and [8] in that the calculation of the textural features is aligned i.e. the window in which the features are extracted moves along the stroke trace, parallel to the stroke boundary. Through this innovation we have several advantages: we can use bigger analysis windows in order to have more texture information, we have more texture information of the border region of the strokes and the method takes the directional nature of the texture formation process into account.

The organization of this paper is as follows. The next section shows the data material used in our work. Section 3 covers the algorithm. In Section 4 experiments and results are given followed by conclusions and an outlook in Section 5 .

\section{Data}

The texture of painted strokes depends primarily on the painting tool and material used. Also the underground affects the appearance of the strokes. But for medieval panel paintings the underground is prepared to be as plain as possible. Thus the effect of the painting underground will be not investigated in this work as well as we have no information about this fact in IR reflectogramms.

The strokes considered in the present study are applied on test panels prepared by a restorer. Bomford specified typical drawing media used for the creation of underdrawings in medieval painted work of art [1]. These drawing media will be examined and considered in this work: graphite, black chalk and silver point are the representatives for the dry strokes and ink applied by brush, quill or reed pen are the considered fluid strokes. Figure 1 gives examples for dry and fluid drawing materials. The first row shows a sample window from a scanned image in the size of $100 \times 80$ pixels. Pixel-value cross-sections along the white line segments from this image can be seen in the second row. The image profile varies clearly between the strokes and the main differences lie within the border regions of the profiles and thus in the border region of the strokes. Hence important information to distinguish between strokes lies within the border region of the strokes and thus even these regions have to be considered 

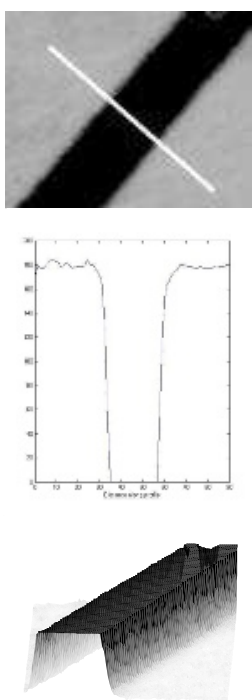

(Quill)
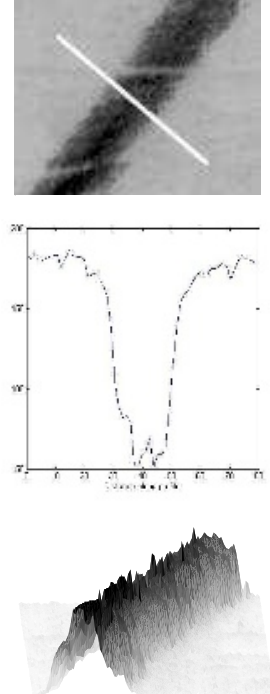

(Graphite)
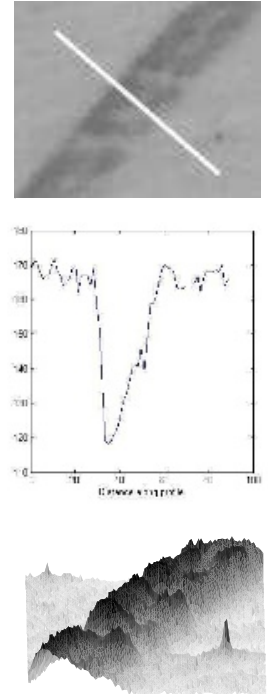

(Silver Point)
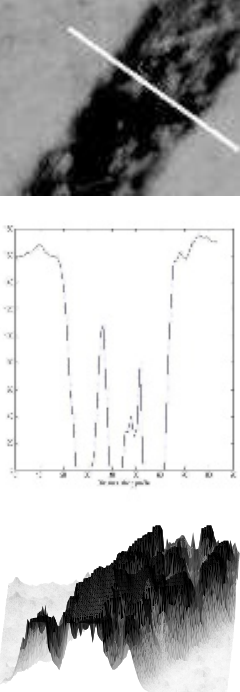

(Black Chalk)

Fig. 1. Strokes considered: normal view, profile and 3D view

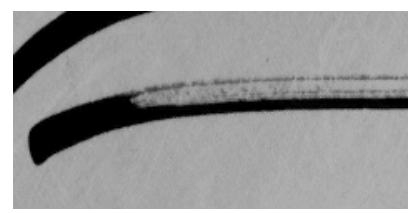

Fig. 2. The reed pen stroke shows discontinuities along the stroke surface (texture)

in the texture analysis. The third row shows a $3 \mathrm{D}$ view of the stroke surface with the pixel value on the $z$-axis. The surface between dry materials varies clearly but the surface from fluid drawing materials is nearby constant. Differences can be seen in the distribution of the texture over the whole stroke. Quill strokes have a very homogeneous black surface. The texture from brush strokes is very similar except to some brighter areas. The surface from the reed pen shows some discontinuities with some brighter areas in the medial part which has less drawing material than the border region. This incident can be seen in Figure 2.

\subsection{Scanned Images}

For our first tests we digitized the panels using a flat-bed scanner with a relative resolution of 1200dpi. Examples of the scanned images can be seen in Figure 1. 


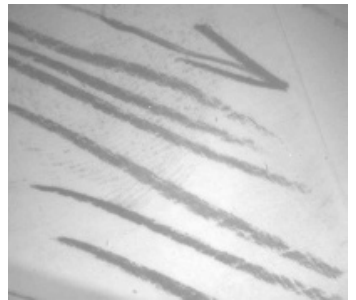

(a)

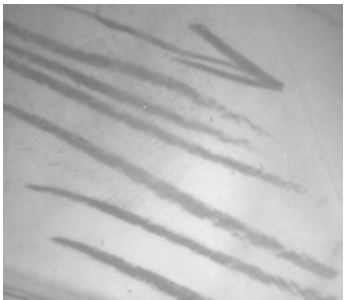

(b)

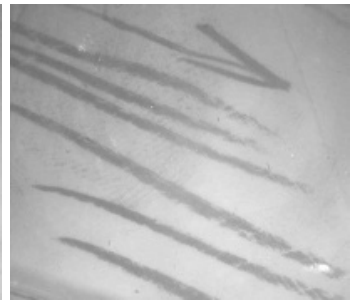

(c)

Fig. 3. IR images without (a) and with the covering color layers Alizarin(b) and Ultramarine blue(c)

\section{$2.2 \quad$ IR Images}

To test our method on real underdrawings we covered the test panels with paint layers in order to simulate real underdrawing strokes. Our acquisition system consists of a Vidicon tube camera (Hamamatsu C1000) with a spectral sensitivity between $900 \mathrm{~nm}$ and $2000 \mathrm{~nm}$ which was attached to a Matrox frame grabber. The size of the digitized images is $629 \times 548$ pixel with an relative resolution of approx. 700 dpi.

The visibility of underdrawing strokes in IR reflectogramms depends on the transmission rate $\tau$ and the thickness of the color pigments and the contrast between underdrawing strokes and the background [9]. For our work we used two color pigments with a high transmission rate. The Schmincke Mussini ${ }^{\circledR}$ colors Alizarin (red) and Ultramarine blue were applied on thin glass plates which covered our test panels in order to simulate underdrawing strokes. Figure 3 shows IR images where the test panels were covered with the paint layers. It can be seen that the strokes and even their texture can be realized in the covered IR images (b) and (c). Only some reflection points and blurred parts can be seen. Remember that the strokes in (b) and (c) cannot be realized in the visible range through the covering paint layer.

\section{Algorithm}

Figure 4 gives an overview of our algorithm. After the acquisition of the test panels and the segmentation of the individual strokes we calculate the medial axis in order to afford the calculation of the textural features along the stroke trace in painting direction. The textural features calculated are the input for the classifier which determines the painting tool and material used for the creation of the stroke.

\subsection{Segmentation}

For our purpose of testing the algorithm on test panels it is sufficient to segment the strokes from the plain background. Through the similarity to document im- 


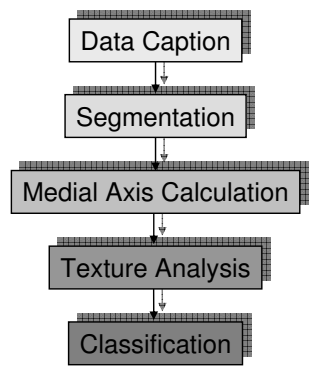

Fig. 4. The overall algorithm for the recognition of painted strokes

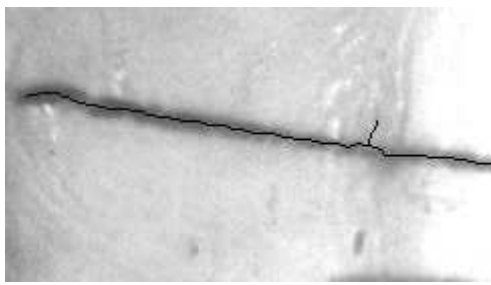

(a)

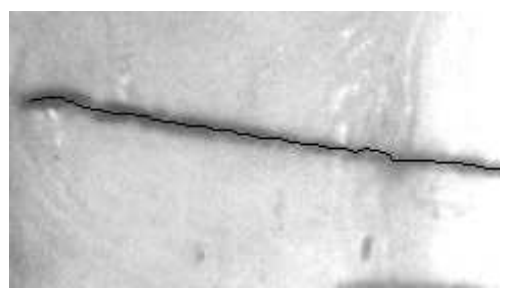

(b)

Fig. 5. (a) Input image, (b) shows a detail from (a) after the segmentation and calculation of the medial axis

age analysis we use the top hat transformation to plain the background followed by a global threshold produced by Otsu's method to segment the strokes from the background. The method showed best result in [7]. Morphological operations remove artifacts from the background and inside the strokes. After segmenting the strokes from the background the intrinsic segmentation of the strokes is done. We calculate the medial axis of the strokes with the thinning algorithm from [15]. To enable the calculation of the textural features a stroke can have only two endpoints of his medial axis. Thus small artifacts from the skeleton are removed by pruning and crossing strokes are separated into individual segments. Figure 5 shows an example of the medial axis with removed artifacts from an IR image.

\section{$3.2 \quad$ Texture Analysis}

The primary task in identifying the painted strokes is the extraction of the textural features. The benefit of our method is the direction controlled analysis. Standard texture analysis algorithms perform parallel to the image boundaries and calculate features for every pixel. For the stroke application it is optimal to scan the textural features along the medial axis of the stroke trace. This condition can be seen in Figure 6 where the rotated sampling window (b) contains more 


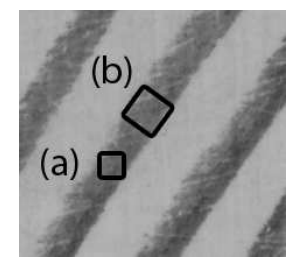

Fig. 6. Sample windows to calculate textural features. (a) standard method, (b) proposed method

texture information than the normal window (a). Through this innovation we have several advantages:

- Bigger sample windows can be adopted to gain the textural features for providing more texture information

- The windows include more border information of the strokes which is fundamental to distinguish between them (see Section 2)

- The performance is better because textural features are calculated only for the segmented part

To gain the textural features we applied two different texture analysis methods. A similar application area is the texture analysis of clouds [2]. So the first method used in our work is the Gray Level Co-occurrence Matrix (GLCM) [5] which showed good results in this work and the second method is the discrete wavelet transformation DWT [10] which also outperformed other methods in several comparative studies $[13,12]$.

Gray Level Co-occurrence Matrix. The GLCM is a very popular tool for texture analysis. It was presented in 1973 by Haralick et al. [5]. The $N \times N$ GLCM describes the spatial alignment and the spatial dependency of the different gray levels, whereas $N$ is the number of gray levels in the original image. The cooccurrence matrix $P_{\phi, d}(i, j)$ is defined as follows. The entry $(i, j)$ of $P_{\phi, d}$ is the number of occurrences of the pair of gray levels $i$ and $j$ at inter-pixel distance $d$ and the direction angle $\phi$. The considered direction angles are $0^{\circ}, 45^{\circ}, 90^{\circ}$ and $135^{\circ}$. For a chosen distance $d=1$ we computed the energy, inertia, entropy and the homogeneity of the mean of these four directions to get a four dimensional feature vector.

Discrete Wavelet Transformation. The discrete wavelet transformation (DWT) [10] decomposes an original signal $f(x)$ with a family of basis functions $\psi_{m, n}(x)$, which are dilations and translations of a single prototype wavelet function known as the mother wavelet $\psi(x)$ :

$$
f(x)=\sum_{n=0}^{\infty} \sum_{m=0}^{\infty} c_{m, n} \psi_{m, n}(x) .
$$

$c_{m, n}$ constitutes the DWT coefficients where $m$ and $n$ are integers and referred to as the dilation and translation parameters. An efficient way to implement this 


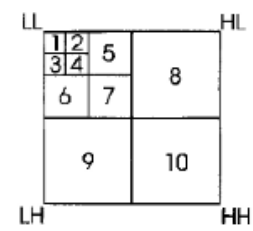

(a)

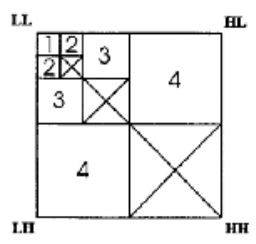

(b)

Fig. 7. (a) 10 channels of a three level wavelet decomposition of an image. (b) Grouping of wavelet channels to form 4 bands to calculate the features [11]

scheme using filters was developed by Mallat [10]. The 2D DWT is computed by a pyramid transform scheme using filter banks. The filter banks are composed of a low pass and a high pass filter and each filter bank is then sampled down at a half rate of the previous frequency. The input image is convolved by a high pass filter and a low pass filter in horizontal direction (rows) followed by another convolution with a high and a low pass filter in vertical direction (columns). Thus the original image is transformed into four sub images after each decomposition step. See [11] for details. A three level decomposition results in 10 sub images, see Figure $7(\mathrm{a})$ whereas the approximation image is the input image for the next level.

We use a Daubechies $d b 10$ motherwavelet for our analysis. The energy of the coefficient magnitudes $c_{m, n}$ is calculated to build a four dimensional feature vector: the HL and LH sub images from each channel are combined and the $\mathrm{HH}$ sub images are not considered because they tend to contain the majority of noise [11], see Figure 7(b).

\section{$4 \quad$ Experiments and Results}

After segmenting the images and the calculation of the medial axis we have 122 scanned strokes and 258 strokes acquired with the Vidicon tube camera. We have 6 classes of strokes from the scanned images and 5 classes for the IR images. Because of the nearly invisibility of the silver point stroke in the IR image they are not considered in this test set. We perform contrast-limited adaptive histogram equalization to enhance the contrast of the IR images.

The number of textural features per stroke depends on its length and the distance between placing the windows. We used a distance of 10 pixels to place the windows along the medial axis of the stroke. To limit the feature space we combined the features calculated within each window by building the mean and standard deviation. Thus we have 8 features for the GLCM method: the mean and standard deviation for the energy, inertia, entropy and homogeneity and 8 DWT features: the mean and standard deviation of the energy in the four channels. The features calculated (GLCM and DWT) were normalized and we used the $k \mathrm{NN}$ classifier to evaluate the drawing tool and material used for the creation of the strokes. 
Table 1. Classification results: method, number of features and percentage of correct classified strokes for scanned and IR images

\begin{tabular}{|l|c|c|c|}
\hline Method & NoF & $(\%)$ SCAN & $(\%)$ IR \\
\hline DWT & 8 & 74.6 & 68.6 \\
GLCM & 8 & 75.4 & 59.3 \\
Combination & 16 & 75.4 & 70.5 \\
\hline
\end{tabular}

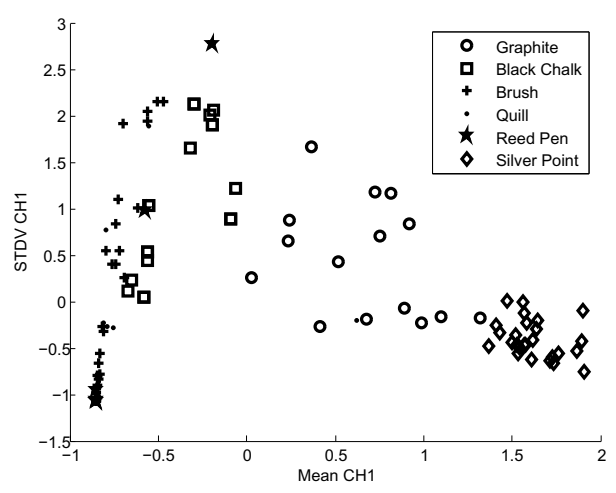

Fig. 8. The feature space from the DWT features in Channel 1

Classification results are tabulated in Table 1 . Best results were obtained with $k=3$. The percentage of correct classified scanned strokes constitutes $74.6 \%$ for the DWT features and $75.4 \%$ for the GLCM features. A combination of the features from both methods could not outperform this results. Expectedly the results for the IR images are a little bit worse due to the limited resolution and contrast. Hence we have $68.6 \%$ for the features from the DWT method and only $59.3 \%$ for the GLCM features. A combination of both brings $70.5 \%$.

To illustrate the visual description in Section 2 we show the feature space of two DWT features in Figure 8 . The $x$-axis shows the mean value of the DWT energy in the first channel and the standard deviation from the energy in the first DWT channel is shown on the $y$-axis. It can be seen that all strokes show compact clusters for their features except the features for the reed pen which are distributed over the whole feature space. Brush, quill and black chalk show low energy values. The quill stroke features cluster in the lower left part of the feature space. They have a very homogeneous black surface and thus low energy and standard deviation. Brush strokes are similar but they have higher standard deviations due to some artifacts in the surface. The black chalk stroke has higher energies and standard deviation due to its coarser texture. Graphite and silver point show increasing values for their energy. Reed pen strokes with its manifold texture are distributed over the whole feature space. As in agreement with the visual description of the strokes in Section 2 fluid materials have low energy values and dry materials like black chalk, graphite and silver point have higher 
Table 2. Confusion Matrix for the Classification results, Scanned images

\begin{tabular}{|l|c|c|c|c|c|c|}
\hline \multirow{2}{*}{$\begin{array}{c}\text { stroke } \\
\text { class }\end{array}$} & \multicolumn{6}{|c|}{ classified as (\%) } \\
\cline { 2 - 7 } & Graphite & Chalk & Brush & Quill & Reed Pen & Silver \\
\hline \hline Graphite & 80.0 & 0.0 & 0.00 & 0.00 & 0.00 & 20.0 \\
\hline Chalk & 10.5 & 89.5 & 0.0 & 0.00 & 0.0 & 0.00 \\
\hline Brush & 0.00 & 16.7 & 50.0 & 33.3 & 0.00 & 0.00 \\
\hline Quill & 4.3 & 0.00 & 34.8 & 60.9 & 0.00 & 0.00 \\
\hline Reed Pen & 0.0 & 12.5 & 75.0 & 12.5 & 0.0 & 0.0 \\
\hline Silver & 0.0 & 0.00 & 0.00 & 0.00 & 0.0 & 100.0 \\
\hline
\end{tabular}

Table 3. Confusion Matrix for the Classification results, IR images

\begin{tabular}{|l|c|c|c|c|c|}
\hline \multirow{2}{*}{$\begin{array}{c}\text { stroke } \\
\text { class }\end{array}$} & \multicolumn{5}{|c|}{ classified as (\%) } \\
\cline { 2 - 6 } & Graphite & Chalk & Brush & Quill & Reed Pen \\
\hline \hline Graphite & 89.1 & 8.7 & 0.00 & 0.00 & 2.2 \\
\hline Chalk & 16.3 & 69.7 & 7.0 & 4.7 & 2.3 \\
\hline Brush & 0.00 & 7.7 & 55.8 & 23.1 & 13.5 \\
\hline Quill & 0.00 & 1.9 & 22.7 & 66.0 & 9.5 \\
\hline Reed Pen & 17.2 & 12.5 & 23.4 & 21.9 & 25.0 \\
\hline
\end{tabular}

energy values. The silver point has the highest energy values which is analog to Figure 1 where the $3 \mathrm{D}$ view from the silver point shows a fine texture with high frequencies.

To show the classification results for the several classes Table 2 and 3 tabulates the confusion matrix for the classification results from the DWT method for the scanned and IR images. The scanned reed pen strokes are distributed over the whole feature space so that no reed pen stroke is classified correct. Best results are within the silver point, the graphite and the black chalk stroke which show compact clusters in Figure 8. Expectedly the dry drawing materials show better results than the fluid materials due to the fact that the texture from the fluid materials is very similar. The confusion matrix for the IR image in Table 3 shows similar results.

\section{Conclusion and Outlook}

In this work a rotational alignment of texture analysis of painted strokes has been discussed. The method developed was able to recognize up to $75 \%$ of painted strokes into a set of 6 predefined classes. We applied two different texture analysis methods, the discrete wavelet transformation and the Gray Level Co-occurrence Matrix. The problems in analyzing the texture of painted strokes, the narrow width and the winding painting trace of the strokes, is avoided by an algorithm which performs along the drawing trace of the strokes to calculate the textural features. So we have a maximum content of stroke texture and we take the 
directional nature of the texture formation process into account. The algorithm can be adopted to the identification of different stroke types in painted work of art as well as the recognition of writing tools in handwritten documents. To improve the classification results we will add profile features to the textural features. Profile classification has been used in the work from [14]. Further more the use of a stronger classifier will be evaluated in our future work.

\section{Acknowledgments}

This work was supported by the Austrian Science Foundation (FWF) under grant P15471-MAT.

\section{References}

1. David Bomford, editor. Art in the Making, Underdrawings in Renaissance Paintings. National Gallery, London, 2002.

2. C. I. Christodoulou, S. C. Michaelides, and C. S. Pattichis. Multifeature texture analysis for the classification of clouds in satellite imagery. IEEE Trans. Geoscience and Remote Sensing, 41(11):2662 - 2668, Nov. 2003.

3. J.V.A. de Boer. Infrared Reflectography.-A contribution to the examination of earlier european paintings. PhD thesis, Univ. Amsterdam, 1970.

4. K. Franke, O. Bünnemeyer, and T. Sy. Ink Texture Analysis for Writer Identification. In 8th International Workshop on Frontiers in Handwriting Recognition, pages 268-273, 2002.

5. R. M. Haralick, K. Shanmugan, and I. Dinstein. Textural Features for Image Classification. IEEE Trans. System Man Cybernetics, 3(6):610-621, November 1973.

6. P. Kammerer, G. Langs, R. Sablatnig, and E. Zolda. Stroke Segmentation in Infrared Reflectograms. In 13'th Scandinavian Conference, SCIA 2003, pages 11381145, Halmstad, Sweden, June 2003. Springer Verlag.

7. G. Leedham, C. Yan, K. Takru, J.H.N.Tan, and L. Mian. Comparison of some thresholding algorithms for text background segmentation in difficult document images. In 7th International Conference Document Analysis and Recognition, pages 859-864, Edinburgh, August 2003.

8. M. Lettner, P. Kammerer, and R. Sablatnig. Texture Analysis of Painted Strokes. In 28th Workshop of the Austrian Association for Pattern Recognition, pages 269276, Hagenberg, Austria, June 2004.

9. Franz Mairinger. Strahlenuntersuchung an Kunstwerken. Buecherei des Restaurators Band 7. E. A. Seemann, 2003.

10. S.G. Mallat. A theory for multiresolution signal decomposition: The wavelet representation. IEEE Trans. Pattern Analysis and Machine Intelligence, 11(7):674-693, July 1989.

11. R. Porter and N. Canagarajah. Robust rotation invariant texture classification. In International Conference Acoustics, Speech, and Signal Procesing ICASSP-97, volume 4, pages 3157 - 3160, Munich, April 1997. 
12. R. Porter and N. N. Canagarajah. Robust relation-invariant texture classification: Wavelet, gabor filter and gmrf based schemes. IEE Proceedings Vision, Image and Signal Processing, 144(3):180-188, June 1997.

13. T. Randen and J.H. Husoy. Filtering for Texture Classification: A Comparative Study. IEEE Trans. Pattern Analysis and Machine Intelligence, 21(4):291-310, April 1999.

14. M. Wirotius, A. Seropian, and N. Vincent. Writer identification from gray level distribution. In 7th International Conference Document Analysis and Recognition, Edinburgh, August 2003.

15. T.Y. Zhang and C.Y. Suen. A fast parallel algorithm for thinning digital patterns. Communications of the ACM, 27(3):236-240, March 1984. 